\title{
Inflation during oscillations of the inflaton
}

\author{
Andrew R. Liddle and Anupam Mazumdar \\ Astronomy Centre, University of Sussex, Falmer, Brighton BN1 9QJ, United Kingdom
}

(Received 10 June 1998; published 14 September 1998)

\begin{abstract}
Damour and Mukhanov have recently devised circumstances in which inflation may continue during the oscillatory phase which ensues once the inflaton field reaches the minimum of its potential. We confirm the existence of this phenomenon by numerical integration. In such circumstances the quantification of the amount of inflation requires particular care. We use a definition based on the decrease of the comoving Hubble length, and show that Damour and Mukhanov overestimated the amount of inflation occurring. We use the numerical calculations to check the validity of analytic approximations. [S0556-2821(98)03020-3]
\end{abstract}

PACS number(s): $98.80 . \mathrm{Cq}$

\section{INTRODUCTION}

Ordinarily, cosmological inflation-a period of accelerated expansion-is considered to be driven by a scalar field $\phi$ rolling slowly, and monotonically, down a shallow potential $V(\phi)$. However, recently Damour and Mukhanov [1] have pointed out that for non-convex potentials, where $d^{2} V / d \phi^{2}$ is negative in regions not too far from the minimum, there exist circumstances where inflation may continue during the oscillations. The basic idea is to arrange that the scalar field spends most of its time on the shallow "wings", of the potential, where it is quite flat, so that for each oscillation there is a period of inflation which overpowers the inevitable non-inflationary region near the core of the potential. One thus obtains on average some inflation over a complete cycle of oscillation. Damour and Mukhanov did not give such behavior a name; we shall call it oscillating inflation.

The oscillations take place on a much shorter time scale than the Hubble expansion. However, over many oscillations the effect of the expansion is felt, and drains energy away from the oscillations. This process continues until the scalar field, known as the inflaton, is trapped completely within the convex core of the potential. There the condition for oscillating inflation fails and inflation ceases in the usual manner.

Because each oscillation is a combination of inflating and non-inflating portions, considerable care is required in defining what is meant by the amount of inflation obtained, and in fact Damour and Mukhanov overestimated the amount of inflation taking place. We discuss in detail the definition of the amount of inflation, and confirm our analytic estimates numerically.

\section{DYNAMICS}

In this section we briefly summarize the results obtained by Damour and Mukhanov [1]. The evolution equations of the scalar field for the flat Friedmann cosmology read

$$
\ddot{\phi}+3 H \dot{\phi}=-\frac{d V}{d \phi} ;
$$

$$
H^{2}=\frac{8 \pi}{3 m_{\mathrm{Pl}}^{2}} \rho,
$$

where $H$ is the Hubble parameter and $\rho \equiv \frac{1}{2} \dot{\phi}^{2}+V(\phi)$ is the energy density of scalar field. Its pressure $p$ is given by $p \equiv \frac{1}{2} \dot{\phi}^{2}-V(\phi)$. From these two further equations can be obtained, which are useful though of course not independent of the first two: namely,

$$
\begin{aligned}
& \dot{\rho}=-3 H(\rho+p)=-3 H \dot{\phi}^{2} ; \\
& \frac{\ddot{a}}{a}=-\frac{4 \pi}{3 m_{\mathrm{Pl}}^{2}}(\rho+3 p) .
\end{aligned}
$$

The effective adiabatic index of the scalar matter can be defined as

$$
\gamma \equiv \frac{\rho+p}{\rho}=\frac{\dot{\phi}^{2}}{\rho} \approx \frac{2}{3} \epsilon_{H},
$$

where $\epsilon_{H}$ is the slow-roll parameter, defined as in Ref. [2], which is required to be less than one for inflation to proceed. Inflation occurs whenever $\gamma<2 / 3$, corresponding to $\rho+3 p$ $<0$.

We assume that initially the scalar field is well displaced from the minimum, and drives a period of slow-roll inflation. We will also follow Damour and Mukhanov in assuming the potential is an even function of $\phi$. As the field approaches the minimum, eventually the slow-roll conditions cease to apply, at some $\phi$ which depends on the nature of the potential. Then rapid oscillations, with frequency $\omega$, dominate the evolution equations. For $\omega \gg H$, one is dealing with two timescales; individual oscillations can be studied ignoring the Hubble expansion, and the effect of the expansion imposed on the behavior averaged over oscillations. Ignoring the Hubble expansion for the time being, the amplitude of the oscillations remains approximately constant with $\rho$ $\approx V\left(\phi_{\mathrm{m}}\right), \phi_{\mathrm{m}}$ being the highest point of oscillation. Equation (1) then reduces to 


$$
\frac{d}{d t}\left(\frac{1}{2} \dot{\phi}^{2}+V(\phi)\right)=0,
$$

and the period $\tau$ of such oscillations is obtained by integrating Eq. (6):

$$
\tau \equiv \frac{2 \pi}{\omega}=4 \int_{0}^{\phi_{\mathrm{m}}} \frac{d \phi}{\sqrt{2\left[V_{\mathrm{m}}-V(\phi)\right]}},
$$

where $V_{\mathrm{m}}=V\left(\phi_{\mathrm{m}}\right)$. We obtain the adiabatic index by averaging over an oscillation

$$
\gamma \equiv\left\langle\frac{\rho+p}{\rho}\right\rangle \equiv\left\langle\frac{\dot{\phi}^{2}}{\rho}\right\rangle .
$$

The condition $\gamma<2 / 3$ can be expressed as

$$
\begin{aligned}
\gamma & =\frac{\left\langle\dot{\phi}^{2}\right\rangle}{V_{\mathrm{m}}}=2\left(1-\frac{\langle V(\phi)\rangle}{V_{\mathrm{m}}}\right) \\
& =2 \frac{\int_{0}^{\phi_{\mathrm{m}}}\left(1-V(\phi) / V_{\mathrm{m}}\right)^{1 / 2} d \phi}{\int_{0}^{\phi_{\mathrm{m}}}\left(1-V(\phi) / V_{\mathrm{m}}\right)^{-1 / 2} d \phi}<\frac{2}{3} .
\end{aligned}
$$

Using the first two equalities one can reduce this inequality to a simpler form

$$
\left\langle V-\phi V_{, \phi}\right\rangle>0
$$

where the comma indicates a derivative.

This has a nice geometric interpretation $[1] ; V-\phi V_{, \phi}$ is the intercept of the tangent to the potential at the point $\phi$, and to obtain inflation on average, then over an oscillation the intercept has to be positive. Note that the above condition fails to be satisfied for power-law potentials $\left(V(\phi) \propto \phi^{q}\right)$ for $q \geqslant 1$. Such potentials must be a good approximation sufficiently close to the minimum of any potential.

\section{QUANTIFYING INFLATIONARY EXPANSION}

In slow-roll inflation, the standard quantification of inflation is the number of $e$-foldings $N$, defined by

$$
N=\ln \frac{a_{\mathrm{f}}}{a_{\mathrm{i}}},
$$

where "i" and "ff" denote initial and final values respectively. However, if slow-roll is not working well this requires modification. This is particularly true for oscillating inflation, where the universe continues to expand during those noninflationary parts of the expansion. They should certainly not count towards the inflationary total, and in fact should count against it.

In such circumstances, the correct definition is to examine the change in the comoving Hubble length [2], $H^{-1} / a$, by defining

$$
\widetilde{N}=\ln \frac{a_{\mathrm{f}} H_{\mathrm{f}}}{a_{\mathrm{i}} H_{\mathrm{i}}} .
$$

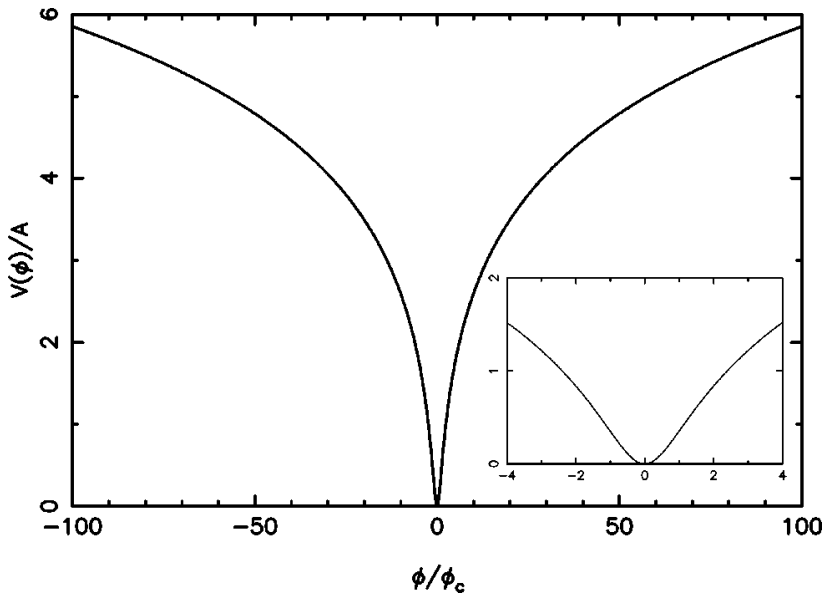

FIG. 1. The potential for $q=0.1$, showing the concave shape for $|\phi| \gg \phi_{\mathrm{c}}$. The inset shows the convex region around the minimum.

There are many reasons why this is the correct general definition. First, the inflationary condition $\ddot{a}>0$ is precisely the condition that $a H$ is increasing. Second, it is the combination $a H$ which determines whether the flatness and horizon problems are being solved. And, most importantly of all, the condition for horizon crossing for a perturbation of comoving wavenumber $k$ is $k=a H$, so it is that combination which decides whether scales are inside or outside the horizon.

In the slow-roll limit $N$ and $\tilde{N}$ are identical, but here they are far from it, and $\widetilde{N}<N$ by definition as $H$ always decreases.

\section{THE DAMOUR-MUKHANOV MODEL}

\section{A. Analytic approximation}

So far the argument holds good for arbitrary potentials. From now on we shall restrict ourselves to potentials which give rise to inflation during oscillations. We consider the one suggested by Damour and Mukhanov [1], which is the potential

$$
V(\phi)=\frac{A}{q}\left[\left(\frac{\phi^{2}}{\phi_{\mathrm{c}}^{2}}+1\right)^{q / 2}-1\right],
$$

where $q$ is a real dimensionless parameter greater than zero. $A$ and $\phi_{\mathrm{c}}$ are dimensionful; $A$ has the dimension of [mass $]^{4}$ and $\phi_{\mathrm{c}}$ is the scale which determines the core of the potential and has the same dimension as $\phi$, namely [mass]. The potential is shown in Fig. 1. It can be reduced to different forms in different regimes. For $q \rightarrow 0, V(\phi)=\frac{1}{2} A \ln \left(1+\phi^{2} / \phi_{\mathrm{c}}^{2}\right)$, while when $\phi_{\mathrm{c}} \ll \phi$ the potential reduces to $V(\phi)$ $\approx A q^{-1}\left(\phi / \phi_{\mathrm{c}}\right)^{q}$. If both limits are taken one gets $V(\phi)$ $\approx A \ln \left(\phi / \phi_{\mathrm{c}}\right)$.

Since oscillating inflation will only occur if the oscillations extend well outside the core region, for most of a cycle the field must obey $\phi \gg \phi_{\mathrm{c}}$ so that the potential reduces to the power-law form. We can approximate the mean behavior by ignoring the core region, an approximation which will hold well until the oscillation amplitude falls close to the core radius and oscillating inflation ceases. For power-law poten- 
tials the adiabatic index $\gamma$ has been computed in Ref. [3] as $\gamma=2 q /(q+2)$ and the various physical quantities evolve as [1]

$$
\begin{aligned}
& a \propto t^{2 / 3 \gamma}=t^{(q+2) / 3 q} \\
& \rho=V\left(\phi_{\mathrm{m}}\right) \propto t^{-2} \propto a^{-6 q /(q+2)} \\
& \phi_{\mathrm{m}} \propto t^{-2 / q} \propto a^{-6 /(q+2)},
\end{aligned}
$$

where $\phi_{\mathrm{m}}$ is the amplitude of the oscillations, $\phi_{\mathrm{c}}<\phi_{\mathrm{m}}$ $<\phi_{\mathrm{s}}, \phi_{\mathrm{s}}$ being the value of $\phi$ where the slow-roll approximation breaks down.

From Eqs. (14)-(16) one can compute Eq. (12)

$$
\tilde{N} \simeq \frac{1-q}{3} \ln \frac{\phi_{\mathrm{s}}}{\phi_{\mathrm{c}}},
$$

where $\phi_{\mathrm{s}}$ is the value of the field at the end of slow roll, which is usually less than $m_{\mathrm{Pl}}$. This is to be contrasted with the amount of expansion

$$
N \simeq \frac{2+q}{6} \ln \frac{\phi_{\mathrm{s}}}{\phi_{\mathrm{c}}} .
$$

Damour and Mukhanov [1] quoted this expression, with the additional approximation $\phi_{\mathrm{s}} \simeq m_{\mathrm{Pl}}$. This latter result is quite misleading; for example it suggests that there is a possibility of enhancement of accelerated expansion due to increase in the value of $q$. Their result is self-contradictory, as we have already seen that there can be no inflation during oscillations for the power-law potentials with $q \geqslant 1$. Our result is supported by the numerical calculation, described in the next subsection.

We also note that the assumption $\phi_{\mathrm{s}} \simeq m_{\mathrm{Pl}}$ is not correct in general. The contrived shape of the potential allows the field to slow roll for a longer period. From Eq. (17), we see that as we approach $q \rightarrow 0$ the prefactor increases and one expects to get higher values of $\widetilde{N}$ for the same values of $\phi_{\mathrm{c}}$. In fact, on the contrary one gets smaller $\widetilde{N}$, because slow roll does not end at $\phi_{\mathrm{s}} \approx m_{\mathrm{Pl}}$ but rather at $\phi_{\mathrm{s}} \approx q m_{\mathrm{Pl}} / \sqrt{16 \pi}$ for $\phi \gg \phi_{\mathrm{c}}$, giving

$$
\tilde{N} \simeq \frac{1-q}{3}\left[\ln \frac{q m_{\mathrm{Pl}}}{\phi_{\mathrm{c}}}-2\right] .
$$

As $q$ approaches zero, $\phi_{\mathrm{s}}$ also goes to zero, implying that the number of $e$-foldings in Eq. (17) also decreases logarithmically. This effect starts dominating as $q \rightarrow 0$ lowering the maximum yield in $\widetilde{N}$, determined by Eq. (19). Hence to obtain the maximum number of $e$-foldings one needs to fix the value of $q$ and then reduce $\phi_{c}$.

For sensible values of the parameters, only a few $e$-foldings of inflation are available. Even if $\phi_{\mathrm{c}}$ is lowered to the electro-weak scale, $\phi_{\mathrm{c}}=10^{-17} m_{\mathrm{Pl}}$, the amount is still limited, for example $q=0.6$ gives $\widetilde{N} \approx 4.9$ and $q=0.1$ gives $\widetilde{N} \approx 10.5$.
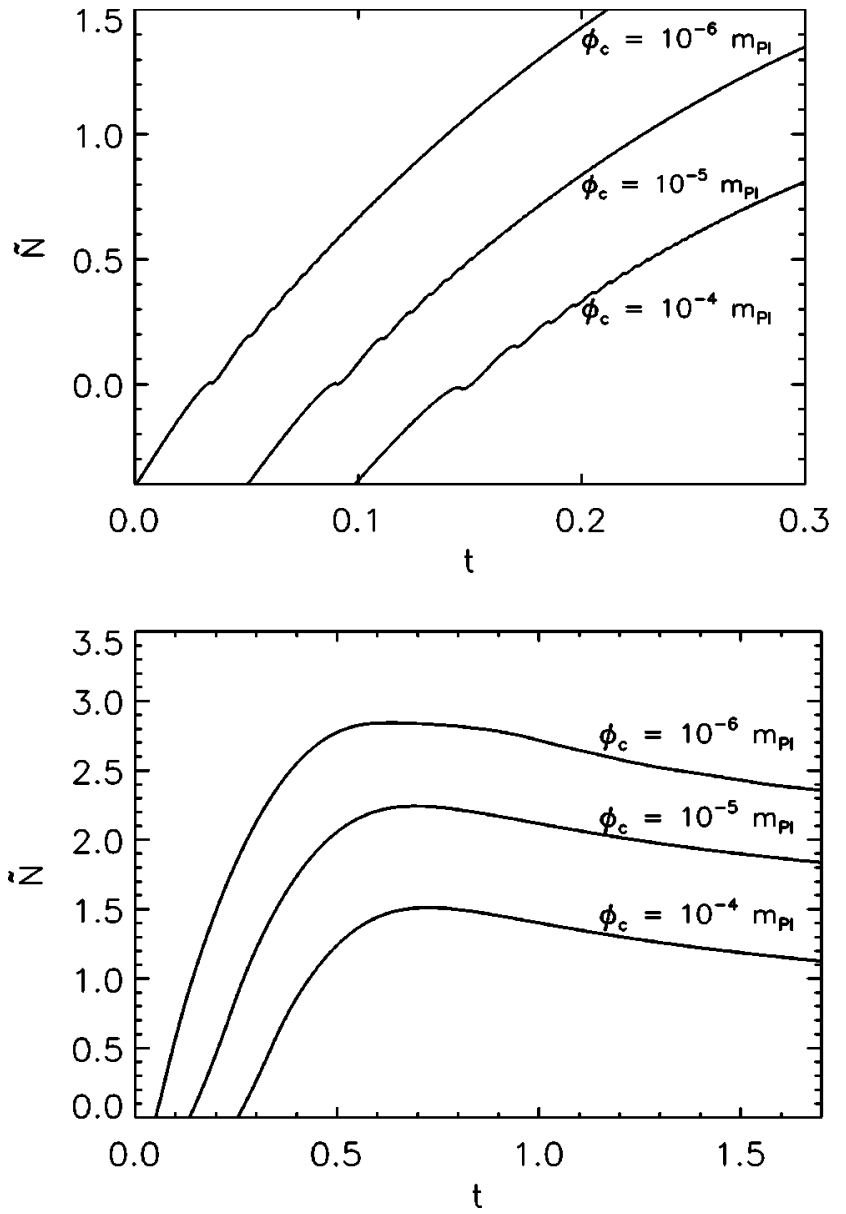

FIG. 2. A numerical simulation of the evolution of $\widetilde{N}$ for $q=0.1$. The upper panel shows the early evolution for two choices of $\phi_{\mathrm{c}}$, showing the last stages of slow-roll and the beginning of the oscillations. The lower panel shows the complete oscillating inflation era for two choices of $\phi_{\mathrm{c}}$; the oscillations are too small to be seen on this scale. In each case, $\widetilde{N}$ is normalized to zero at the end of slow-roll inflation, and an arbitrary horizontal shift has been used to separate the lines. The heights of the maxima in the lower panel are accurately given by Eq. (19).

For a logarithmic potential the adiabatic index $\gamma$ $=1 / \ln \left(\phi_{\mathrm{m}} / \phi_{\mathrm{c}}\right)[1]$, and we are unable to give a simple form of $\widetilde{N}$ for such a potential.

\section{B. Numerical analysis}

In this section we describe our numerical results. We consider the potential of Eq. (13) and evolve the field equations numerically. In Figs. 2 and 3 we depict the evolution of $\widetilde{N}$ for two different values of $q$. The first hump describes the end of slow-roll approximation, which is where oscillating inflation is considered to start. For normal potentials there is a sharp decrease in the value of $\widetilde{N}$ after slow-roll inflation ends, but for the potential under consideration we see further $e$-foldings; each oscillation has inflationary and noninflationary parts but the former dominate leading to a continued upward trend. Eventually, as seen in the lower panel of Fig. 2, the field finds its way to the true core and oscillat- 


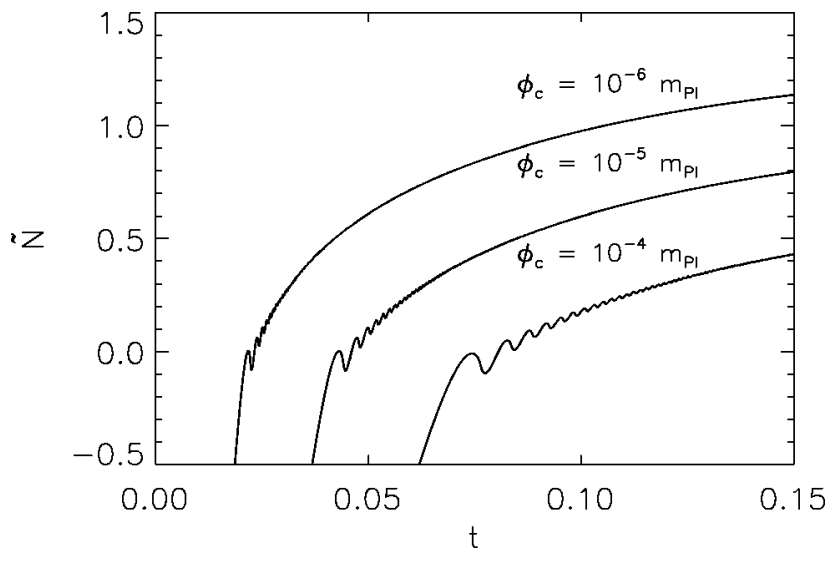

FIG. 3. As the upper panel of Fig. 2, but for $q=0.6$.

ing inflation ends. Its total amount is measured by the maximum height of $\widetilde{N}$ above its value at the end of slow-roll inflation.

Figure 4 compares our analytic estimate Eq. (19) of the amount of inflation with our numerical results, as a function of $q$. The agreement is extremely good. There is no inflation for $q>1$, and as $q$ is decreased the amount of inflation increases almost linearly before reaching its maxima and then starts decreasing as $q$ approaches zero. In this last regime the analytic approximation fails to work well, with the potential approaching the logarithmic limit as explained in the previous section. For small values of $q$ we are unable to compare the numerical results as we have not been able to derive any analytical expression.

\section{DENSITY PERTURBATIONS}

As well as solving problems of initial conditions, inflation plays a crucial role in generating density perturbations which can later seed structure formation. Because the oscillating inflation is brief, the observed perturbations are normally expected to originate in the slow-roll regime which precedes the oscillations, though in principle a subsequent additional period of inflation could push them to observable scales [1]. In this short section, we check that required amplitude of density perturbations generated in the slow-roll epoch does not give an uncomfortable constraint on the potential under consideration.

Defining the primordial density perturbation spectrum $\delta_{\mathrm{H}}$ as in Ref. [4], one has

$$
\delta_{\mathrm{H}}^{2} \approx \frac{32}{75} \frac{V}{m_{\mathrm{Pl}}^{4}} \frac{1}{\epsilon},
$$

where the slow-roll parameter $\epsilon$ is given by

$$
\epsilon=\frac{m_{\mathrm{Pl}}^{2}}{16 \pi}\left(\frac{V, \phi}{V}\right)^{2} .
$$

The perturbations observed by the Cosmic Background Explorer $(\mathrm{COBE})$ satellite require $\delta_{\mathrm{H}} \approx 2 \times 10^{-5}$; since the

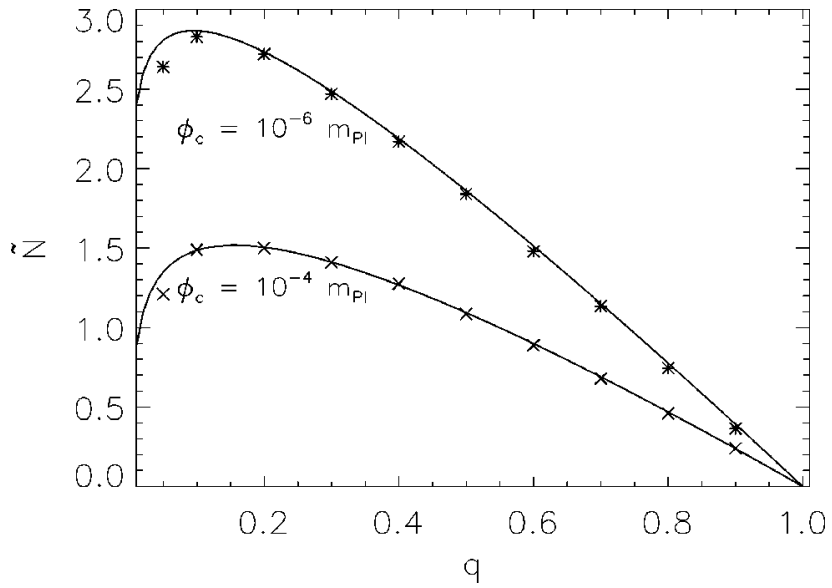

FIG. 4. A comparison of numerical and analytical solutions of the number of e-foldings for $\phi_{\mathrm{c}}=10^{-4} \mathrm{~m}_{\mathrm{Pl}}$ and $10^{-6} \mathrm{~m}_{\mathrm{Pl}}$. The symbols correspond to the numerical results, and the smooth curves to the analytical estimate of Eq. (19).

number of $e$-foldings during the oscillating inflation phase is small, these perturbations must be generated during the slowroll phase. This phase ends when $\epsilon \approx 1$, which as commented above is at

$$
\phi_{\mathrm{s}} \approx \frac{q}{\sqrt{16 \pi}} m_{\mathrm{Pl}} \text {. }
$$

During the slow-roll phase the power-law approximation to the potential is an excellent one and yields

$$
\delta_{\mathrm{H}}^{2}=\frac{512 \pi}{75} \frac{A}{q^{3} m_{\mathrm{Pl}}^{6}} \frac{\phi^{2+q}}{\phi_{\mathrm{c}}^{q}} .
$$

The value of the amplitude $A$ of the potential is to be adjusted to give the right level of perturbations when our present Hubble scale crossed outside the horizon during inflation, of order $50 e$-foldings before inflation finally ends. The amount of inflation in the slow-roll epoch can be adequately computed using the normal slow-roll formula

$$
N \simeq-\frac{8 \pi}{m_{\mathrm{Pl}}^{2}} \int_{\phi}^{\phi_{\mathrm{s}}} \frac{V}{V_{, \phi}} d \phi
$$

and approximating $\tilde{N} \simeq N$. We assume the perturbations are generated $50 e$-foldings from the end of inflation, and ignore the oscillating inflation contribution as negligible given the uncertainty in this number. We then find, for example, that with $q=0.1$ and $\phi_{\mathrm{c}}=10^{-6} m_{\mathrm{Pl}}$, the required amplitude of the potential is $A^{1 / 4} \simeq 5 \times 10^{-3} m_{\mathrm{Pl}}$. This is a fairly typical sort of number for inflationary models. We conclude that the appropriate level of density perturbations can be readily achieved in these models. 


\section{SUMMARY}

We have analyzed the possibility of oscillating inflation both analytically and numerically. A more accurate quantification of the amount of inflation shows that it was overestimated by Damour and Mukhanov [1], and we have given a more appropriate definition which is in excellent agreement with our numerical analysis. Only a few $e$-foldings of oscillating inflation are possible.

\section{ACKNOWLEDGMENTS}

A.R.L. was supported by the Royal Society and A.M. by the INLAKS foundation and ORS. A.R.L. thanks the Fermilab astrophysics group, CfPA Berkeley and the University of Illinois at Urbana-Champaign for hospitality while part of this work was done. We acknowledge use of the Starlink computer system at the University of Sussex.

7222 (1994)

[3] M. S. Turner, Phys. Rev. D 28, 1243 (1983).

[4] A. R. Liddle and D. H. Lyth, Phys. Rep. 231, 1 (1993).

[2] A. R. Liddle, P. Parsons, and J. D. Barrow, Phys. Rev. D 50, 